\title{
GIMNASIA RÍTMICA: PERFIL SOBRE LA PRODUCCIÓN CIENTÍFICA EN REVISTAS DE LATINOAMÉRICA, CARIBE Y PAÍSES IBÉRICOS
}

\author{
Leticia Cristina Lima Moraes ${ }^{1}$, Marcelo Moraes e Silva ${ }^{1}$, leda Parra Barbosa Rinaldi², \\ Jeferson Roberto Rojo' ${ }^{2}$, Leonardo do Couto Gomes ${ }^{1}$ \\ (1) Universidad Federal de Paraná (2) Universidad Estadual de Maringá
}

\begin{abstract}
Envío original: 2018-06-04. Reenviado: 2018-11-15, 2018-12-11. Aceptado: 2018-12-28.
Publicado en versión en español: 2019-07-17
\end{abstract}

Resumen: La presente investigación tiene como objetivo mapear la producción del conocimiento sobre la Gimnasia Rítmica publicada en revistas de Latinoamérica, Caribe y de los países ibéricos. Para cumplir este fin, se eligió la propuesta de cuño exploratorio descriptivo, enlistando los artículos catalogados en las siguientes bases de datos: Scielo, Lilacs y Medline, accedidos a través de la interfaz de la Biblioteca Virtual de la Salud y Redalyc. Al finalizar, el estudio encontró 48 artículos publicados en 27 revistas diferentes, distribuidos en el período de 2001-2017. La temática que más despertó interés en los investigadores es referente al Entrenamiento, que correspondió al 41,67\%. A pesar del predominio del referido eje, se encontró una notable pluralidad epistemológica que contempla: aspectos psicológicos, educativos y sociales, salud, culturales e históricos. Se concluye, por lo tanto, la existencia de un escenario auspicioso para un área que busca consolidarse científicamente, siendo necesario el crecimiento de estudios amparados en las demás formas de plantear el deporte, ascendiendo esas producciones de manera cuantitativa y cualitativa.

Palabras claves: Gimnasia Rítmica; Producción del Conocimiento; Revistas.

\section{Introducción}

La Gimnasia Rítmica (GR) se originó a mediados de 1930 y alcanzó su reglamentación y reconocimiento por el COI (Comité Olímpico Internacional), pero como deporte olímpico solo hasta 1984, y, debido a eso, su primera participación como modalidad competitiva solamente en los Juegos de Los Ángeles 1984 (Santos, Lorenço \& Gaio, 2010). Se destaca que esta modalidad de gimnasia tiene dos tipos de pruebas, las individuales y las de conjunto, y que en 1984 únicamente las pruebas individuales formaron parte del programa de los Juegos Olímpicos, por otra parte, las pruebas de conjunto no se incluyeron sino hasta en 1996 en los Juegos Olímpicos de Atlanta (USA). Por consiguiente, se entiende que como deporte la GR es relativamente joven, puesto que se convirtió en modalidad olímpica hace apenas tres décadas. 
Con respecto a las competiciones, en la actualidad, los países que más se destacan a nivel mundial son los países de la antigua Unión Soviética y Bulgaria, entre otros del este europeo (Lourenço, 2015). El Caribe y los Países Iberoamericanos solo ganaron notoriedad en la esfera mundial en los Juegos Olímpicos de Barcelona en 1992, con una medalla de plata española en el individual general, y en Atlanta 1996, con el título olímpico en las pruebas de conjunto también de la selección hispana. En el caso de Latinoamérica, Lourenço (2003) demuestra que solamente a partir de 1999, en los XXII Juegos Panamericanos en Winnipeg (Canadá), edición en la que Brasil fue campeón, fue cuando la región alcanzó resultados significativos a nivel internacional, lo que trajo la visibilidad de la GR a esa parte del globo y, como consecuencia, nuevos jugadores potenciales de la modalidad. Hasta el presente momento, la participación de los países caribeños es simbólica, a pesar de que países como Cuba y Puerto Rico también están presentes en las grandes competiciones mundiales. Se observa, por lo tanto, un escenario, en cierto modo, prometedor para el desarrollo de la referida modalidad en el contexto caribeño, latino e ibero americano, lo que vuelve importante evidenciar cómo los investigadores de estas localidades están explorando la Gimnasia Rítmica.

En ese sentido, surge el interés en investigar cómo esta modalidad deportiva está siendo estudiada en Latinoamérica, el Caribe y los países ibéricos. Por lo tanto, el presente artículo buscó responder a la siguiente problemática de investigación: ¿Cuál es el perfil de la producción científica sobre la gimnasia rítmica en revistas de Latinoamérica, el Caribe y los Países Ibéricos? El objetivo general consiste en trazar un panorama de la producción científica escrita en revistas de Latinoamérica, Caribe y los Países Ibéricos, sobre la modalidad Gimnasia Rítmica. Los objetivos específicos son: a) verificar el perfil temático de las producciones sobre la gimnasia rítmica en Latinoamérica, el Caribe y los Países Ibéricos, b) evidenciar las autorías / coautorías e instituciones, así como sus redes de colaboración.

\section{Metodología}

Para la composición del alcance de la investigación se eligieron artículos indexados en las siguientes bases de datos: Scielo (Scientific Electronic Library Online), Lilacs (Literatura Latinoamericana y del Caribe en Ciencias de la Salud) y Medline (Medical Literature Analysis and Retrievel System Online) accedidos a través de la interfaz de la Biblioteca virtual de la Salud (BVS-BIREME) y Redalyc (Red de Revistas Científicas de Latinoamérica y el Caribe, España y Portugal). Esas bases fueron seleccionadas por presentar contenido integral en relación a las indexaciones de 
diferentes revistas que son de diversificadas áreas del conocimiento y contemplan diferentes naciones de Latinoamérica, del Caribe y de los Países Ibéricos.

Se utilizaron descriptores en tres idiomas: español, inglés y portugués, y los términos utilizados fueron los siguientes: "gimnasia rítmica", "gimnasia rítmica deportiva", "Sports Rhythmic Gymnastics", "Rhythmic Gymnastics", "Gimnasia Rítmica", "Gimnasia Rítmica deportiva". Con la finalidad de investigar las producciones más antiguas sobre la temática, la temporalidad no fue restringida, es decir, fueron seleccionados artículos desde el primer año encontrados en las bases enlistadas, hasta agosto de 2017, período en el cual se realizó la recolección de los materiales, restringiendo, por lo tanto, el presente estudio al recorte temporal de 2001 a 2017. En cuanto a la exclusión, los artículos duplicados y/o encontrados en más de una base de datos fueron considerados solamente una vez, totalizando al final 48 artículos.

La catalogación se hizo en una hoja de cálculo y se utilizó la siguiente información contenida en los artículos: a) autores; b) país de la institución; c) año de publicación; d) revista; e) temática central del artículo. La información relacionada con los autores no localizados en el propio artículo fue recolectada en el Currículo Lattes $^{1}$ y en las páginas en línea de las universidades a las cuales se vinculan los investigadores. Se vuelve importante resaltar la vulnerabilidad de la catalogación en la información de algunos autores, ya que no están registrados en plataformas de fácil acceso, lo que dificulta la adquisición de su información profesional.

Con el fin de identificar los enfoques temáticos de los artículos, se realizó una lectura preliminar y/o completa de los trabajos para después, en base al estudio de Souza, Moraes e Silva y Moreira (2016), organizarlos en categorías que agruparan los temas encontrados. Para ello, fue necesaria la clasificación en más de un eje, considerando la correspondencia simultánea de determinados trabajos con diferentes temáticas. Los artículos encontrados se clasificaron dentro de los siguientes ejes temáticos:

- Entrenamiento: espacios y equipos relacionados con el desempeño, aspectos motores, fisiológicos, técnicos, tácticos y biomecánicos referentes al rendimiento; Currículos, de Grupos de investigación e Instituciones en un único sistema de información. El Currículum Lattes, por lo tanto, es un estándar brasileño de registro de información de los estudiantes e investigadores del referido país, adoptado por la mayoría de las instituciones de fomento, universidades e institutos de investigación de Brasil. 
- Iniciación Deportiva y Categorías de Base: metodología de enseñanza, aprendizaje motor, entrenamiento de jóvenes atletas y selección de talentos deportivos;

- Salud: promoción, mantenimiento y rehabilitación de la salud, lesiones y patologías;

- Aspectos sociales, culturales e históricos del deporte: aspectos sociológicos, antropológicos e históricos relacionados con el deporte (discusiones sobre temáticas tales como género, medios de comunicación, mercadeo, violencia, valores, etc.);

- Aspectos Nutricionales: perfil dietético y suplementación alimentar.

Para la exposición de los resultados de la investigación, primero se presentan los números generales y se especifica la cantidad de publicaciones sobre la gimnasia rítmica en orden cronológico, y su distribución en las revistas. Luego, se evidencian los autores e instituciones con mayor productividad, y, por fin, se hace un desglose de los asuntos abordados en cada uno de los ejes temáticos.

\section{Resultados y discusión}

Con base en los datos obtenidos, se presenta la distribución de los 48 artículos encontrados durante la periodicidad constatada (2001-2017), conforme la tabla a continuación:

Tabla 1

Número de artículos publicados por año - Sistematizado por los autores.

\begin{tabular}{ccc}
\hline Año & Total & $\%$ \\
\hline 2001 & 1 & $2,08 \%$ \\
\hline 2005 & 1 & $2,08 \%$ \\
\hline 2006 & 3 & $6,25 \%$ \\
\hline 2007 & 1 & $2,08 \%$ \\
\hline 2009 & 3 & $6,25 \%$ \\
\hline 2010 & 4 & $8,33 \%$ \\
\hline 2011 & 2 & $4,17 \%$ \\
\hline 2012 & 7 & $14,58 \%$ \\
\hline 2013 & 6 & $12,50 \%$ \\
\hline 2014 & 5 & $10,42 \%$ \\
\hline 2015 & 6 & $12,50 \%$ \\
\hline 2016 & 8 & $16,67 \%$ \\
\hline 2017 & 1 & $2,08 \%$ \\
\hline
\end{tabular}


El año 2016 fue el año con mayor número de publicaciones, correspondiendo al 16,67\% (ocho artículos), luego aparece el año 2012 con siete artículos, y 2013 y 2015, ambos con un total de seis publicaciones. Como se puede observar, existe una brecha entre los años 2001 y 2005, períodos que también presentan baja producción acerca de la modalidad. Sin embargo, la distribución es relativamente proporcional en cada año, con un alza a partir del año 2012. En relación con la baja producción verificada en 2017, se presupone el retraso en las publicaciones de sus volúmenes, lo que compromete la cronología de la publicación o el caso de las revistas que todavía no disponen sus artículos en esas bases hasta el período final de la catalogación.

Para evidenciar las publicaciones y su distribución en las revistasen cuestión, la tabla siguiente muestra la cantidad de artículos publicados por cada revista:

Tabla 2

Revistas que publicaron artículos sobre gimnasia rítmica - Sistematizado por los autores

\begin{tabular}{ccc}
\hline REVISTA & PAÍS & $\begin{array}{c}\text { NÚMERO DE } \\
\text { ARTícULOS }\end{array}$ \\
\hline Revista Brasileña de Educación Física y Deporte & Brasil & 6 \\
\hline Revista Brasileña de Medicina del Deporte & Brasil & 5 \\
\hline Revista Brasileña de Ciencia y Movimiento & Brasil & 4 \\
\hline Pensar la Práctica & Brasil & 3 \\
\hline Revista Brasileña de Ciencias del Deporte & Brasil & 2 \\
\hline Motriz & Brasil & 2 \\
\hline International Journal of Morphology & Chile & 2 \\
\hline Acta Scientiarum Health Sciences & Brasil & 2 \\
\hline Revista de la Educación Física (Uem. Online) & Brasil & 2 \\
\hline Fitness \& Performance Journal & Brasil & 2 \\
\hline Revista Andaluza de Medicina del Deporte & España & 2 \\
\hline Suma General & & 32 \\
\hline
\end{tabular}

En la tabla 2 se encuentran las 11 revistas que publicaron más de un artículo sobre la temática; 16 revistas publicaron solamente un artículo. La Revista Brasileña de Educación Física y Deporte tiene la cantidad más grande de manuscritos, contabilizados seis artículos. En secuencia, la Revista Brasileña de Medina del Deporte con cinco textos, la Revista Brasileña de Ciencia y Movimiento con cuatro manuscritos y la revista Pensar la Práctica con tres artículos. A continuación, aparecen las revistas con apenas 
dos artículos en sus páginas: Revista Brasileira de Ciencias del Deporte, Motriz, International Journal of Morphology, Acta Scientiarum Health Sciences, Revista de Educación Física, Fitness \& Performance Journal y Revista Andaluza de Medicina del Deporte.

Cabe resaltar que, de un total de 27 revistas, siete son españolas con un total de ocho publicaciones referentes al tema (Journal of Human Sport and Exercise, Nutrición Hospitalaria, Revista de Investigación, Motricidad. European Journal of Human Movement, Revista de Psicología del Deporte, Revista Iberoamericana de Psicología del Ejercicio y el Deporte, Revista Andaluza de Medicina del Deporte). Apenas una es colombiana con un estudio (Revista de Salud Pública), una mexicana con un artículo (Ra Ximhai) y una chilena con dos publicaciones (International Journal of Morphology). Esas revistas contabilizan un total de 12 manuscritos sobre la modalidad, mientras que las revistas brasileñas (17) se componen de 36 textos, lo que evidencia la predominancia de Brasil con relación a la cantidad de revistas y publicaciones.

Esa discrepancia respecto a las producciones, también se puede notar al observar a cuáles instituciones los autores estaban vinculados y sus respectivas nacionalidades, como indica el gráfico abajo:

\section{Tabla 3}

Países de las instituciones, a las cuales estaban vinculados los autores que produjeron sobre la GR (de acuerdo con la información obtenida del respectivo artículo / de las plataformas en línea).

\begin{tabular}{ccc}
\hline $\begin{array}{c}\text { PAÍS DE LA } \\
\text { INSTITUCIÓN }\end{array}$ & TOTAL & $\%$ \\
\hline Brasil & 35 & $66,04 \%$ \\
\hline España & 8 & $15,09 \%$ \\
\hline Venezuela & 2 & $3,77 \%$ \\
\hline Portugal & 2 & $3,77 \%$ \\
\hline México & 1 & $1,89 \%$ \\
\hline Francia & 1 & $1,89 \%$ \\
\hline Cuba & 1 & $1,89 \%$ \\
\hline Canadá & 1 & $1,89 \%$ \\
\hline Chile & 1 & $1,89 \%$ \\
\hline Estados Unidos & 1 & $1,89 \%$ \\
\hline
\end{tabular}

Brasil presenta un número significativo de 35 (64,04\%) instituciones vinculadas a los investigadores que publicaron sobre el tema. Luego, España con ocho instituciones, y Portugal y Venezuela con dos instituciones. Con solo una institución, aparecen los siguientes países: Francia, Cuba, México, Canadá, Chile y Estados Unidos. 
Los países situados fuera de la delimitación espacial establecida para este artículo, es decir, Francia, Canadá y Estados Unidos, poseen autores vinculados a una institución cada uno, pero la publicación de sus trabajos sobre la gimnasia rítmica fue realizada en revistas brasileñas. Con la publicación en la Revista Brasileña de Educación Física y Deporte, la investigadora Monique Loquet, está vinculada a la Universidad de Rennes, en Francia (Loquet, 2016). El brasileño Marcos Alencar Abaide Balbinotti, coautor del texto publicado en la revista Pensar la Práctica, es docente vinculado a la Universidad de Quebec en Trois-Rivières - Canadá (Fontana, Barbosa, Balbinotti \& Balbinotti, 2013). Por último, la Revista Brasileña de Ciencia y Movimiento tiene en sus páginas el manuscrito de la también brasileña Priscila Martins Caçola, vinculada a Texas A \& M University, ubicada en los Estados Unidos (Caçola \& Ladewig, 2007).

El siguiente análisis discurre sobre los autores y coautores que más produjeron sobre la modalidad.

Tabla 4

Los 6 principales autores y coautores con más de una publicación - Sistematizado pelos autores.

\begin{tabular}{ccc}
\hline Autores & Número de artículos & Países de los autores \\
\hline Lenamar Fiorese Vieira & 4 & Brasil \\
Luciana de Souza Menezes & 3 & Brasil \\
Rodrigo Franco de Oliveira & 3 & Brasil \\
Andre Wilson de Oliveira Gil & 3 & Brasil \\
Jose Fernandes Filho & 3 & Brasil \\
Jose Luiz Lopes Vieira & 3 & Brasil \\
\hline
\end{tabular}

Lenamar Fiorese es la investigadora con el número más grande de artículos publicados. La referida autora es docente de la Universidad Estatal de Maringá, institución en la que actúa también como docente del Programa de Postgrado Asociado en Educación Física - Universidad Estadual de Maringá / Universidad Estadual de Londrina. Las líneas de investigación desarrolladas por la investigadora son las siguientes: aprendizaje y desarrollo motor, aspectos psicopedagógicos de la Educación Física y del Deporte, deporte y desempeño motor. Su forma de plantear el objeto de investigación la hereda de su formación en Educación Física en 1982 y Psicología en 2010. Igualmente, posee una maestría (1993) y un doctorado (1999) en Educación Física. Además de su carrera académica, es imprescindible mencionar su relación directa con la esfera de la GR, ya que desde el 2000 es asesora de la selección brasileña de la modalidad, vínculo que puede haber contribuido para la predominancia de sus 
producciones sobre el tema, publicando un manuscrito en cada una de las siguientes revistas: Acta Scientarum Health Sciences (Vieira, Botti \& Vieira, 2005), Motriz (Vieira, Vieira, Amorim, Amorim \& Rocha, 2009), Revista Brasileña de Medicina del Deporte (Vieira, Vieira, Amorim, Amorim \& Rocha, 2009) y Pensar la Práctica (Nakashima, Nascimento Junior \& Vieira, 2012).

Seguidamente, aparece Luciana de Souza Menezes, con tres artículos. Máster graduada por la Universidad Castelo Branco en 2004 y Doctorado en Ciencias del Deporte de la Universidad de Trás-os-Montes y Alto Douro, Portugal (2012), tiene una trayectoria estrecha con el deporte. Su disertación trata sobre aspectos biológicos de atletas de GR y su tesis sobre el perfil morfológico de gimnastas. Actualmente es docente adjunta del Instituto de Educación Física y Deportes de la Universidad del Estado de Río de Janeiro. Sus artículos se encuentran en las siguientes revistas: Fitness \& Performance Journal (Menezes \& Fernandes Filho, 2006), Revista de Salud Pública (Menezes, Novaes \& Fernandes Filho, 2012), International Journal of Morphology (Menezes, Novaes \& Fernandes Filho, 2014).

El profesor José Fernandes Filho tiene una maestría, obtenida en 1989 en la Universidad de Ribeirão Preto y un doctorado también en Educación Física por el Instituto de Investigación Científica de Cultura Física y Deportes de Rusia en 1997. El investigador está vinculado a la Escuela de Educación Física y Deporte de la Universidad Federal de Río de Janeiro y del Programa de Postgrado en Educación Física de la misma institución. Él también produjo tres artículos junto con Luciana de Souza Meneses, a quien orientó en la maestría.

También con tres artículos está Rodrigo Franco de Oliveira. El investigador es graduado en Fisioterapia de la Facultad Claretiana de Batatais (1988), máster en Educación Física por la Universidad Católica de Brasilia (2001) y doctor en Ingeniería Biomédica por la Universidad del Valle del Paraíba (2008). Actualmente es docente del Centro Universitario de Annapolis, y miembro de la comisión para la cual se encargó de la elaboración e implantación del Curso de Postgrado Stricto Sensu y Maestría Profesional en el Ejercicio Físico en la Promoción de la Salud, en el área de Educación Física, de la Universidad Norte de Paraná. Las respectivas producciones son resultados de coautorías, contabilizando un texto en cada una de las siguientes revistas: Unopor Científica Ciencias Biológicas y de la Salud (Guiotte et al, 2012), en la Revista Brasileira de Medicina del Deporte (Lamb et al., 2014) y en la revista Conscientiae Saúde (Hirata \& Oliveira, 2015).

El investigador André Wilson de Oliveira Gil, tiene coautoría en tres artículos, dos de ellos junto con Rodrigo Franco de Oliveira y de uno más en la Revista Brasileña de Medicina del Deporte (Shigaki et al., 2013). Gil es graduado por la Universidad Norte de 
Paraná (2011) y máster (2014) en Fisioterapia (Unopar). Actualmente es doctorando en el Programa de Educación Física UEM / UEL.

El último investigador con tres manuscritos es José Luiz Lopes Vieira, quien tiene una maestría (1993) y un doctorado (1999) por la UFSM en Educación Física. Vieira es docente en la Universidad Estatal de Maringá y en el Programa de Postgrado en Educación Física UEM / UEL. Sus líneas de investigación se relacionan con el desarrollo motor y la psicología del deporte y del ejercicio. Actualmente es Editor del Journal of Physical Education (antigua Revista de la Educación Física / UEM). En cuanto a los trabajos sobre la modalidad, fueron productos de investigaciones colectivas realizadas con Lenamar Fiorese Vieira los artículos publicados en la revista Motriz (Vieira, Vieira, Amorim, Amorim \& Rocha, 2009) y en la Revista Brasileña de Medicina del Deporte (Vieira, Vieira, Amorim, Amorim \& Rocha, 2009) y en la Acta Scientarum Health Sciences (Vieira, Botti, Y Vieira, 2005).

Al analizar las autorías y coautorías se percibe una intensa red de colaboración, dado que los autores que tienen la mayor cantidad de artículos sobre la GR los producen de manera conjunta. En el gráfico siguiente, se puede observar esa tendencia de producción en alianza:

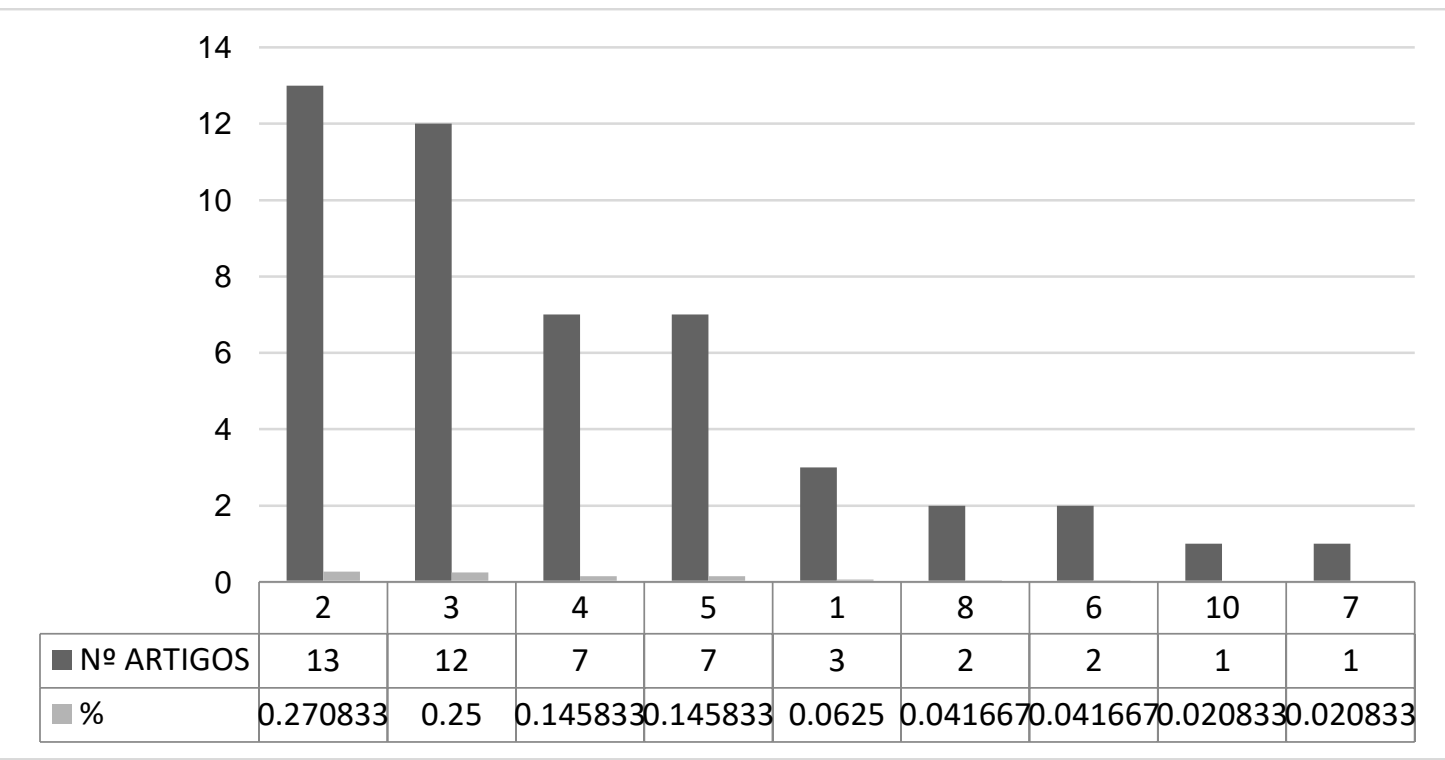

Gráfico 1: Número de autores y coautores por artículo - Sistematizado por los autores

Se encontraron apenas tres artículos con un único autor, siendo el 6,25\% de todos los manuscritos catalogados, y los mismos son de autorías diferentes. El predominio de la red de colaboración entre investigadores en los artículos es notable, puesto que 13 producciones fueron realizadas por dos autores, y luego 12 escritos por 
tres investigadores. Con cuatro autores hay la correspondencia de siete manuscritos, así como la asociación de cinco colaboradores que también contabilizan siete. Dos textos con ocho autores, y otros dos con seis involucrados. Por último, hay un artículo con seis miembros, y otro que contiene la participación de 10 investigadores. Esa tendencia de publicar de forma conjunta se puede explicar, según apunta García, Martrucelli, Rossillo, Denardin, (2010), por un mayor crédito, costeo de investigaciones y legitimación dentro del medio académico. A fin de cuentas, la materialización de un capital en esa esfera ocurre por medio de la producción científica, y por lo tanto la forma más recurrente de obtener una eficiencia de esa productividad proviene de la asociación entre investigadores, principalmente de las denominadas coautorías.

Leta y Cruz (2003) indican que las coautorías pueden ser indiciadoras de asociación científica entre países, instituciones e investigadores. Según García et al. (2010) estos científicos tienen por objetivo diversos y complejos propósitos, que van más allá de la expansión del conocimiento científico, pues permiten que el investigador pueda tener su producción evaluada, ya que la autoría se usa como criterio para la concesión de recursos por las agencias de fomento, además de ser una herramienta de evaluación de los cursos de postgrado y un parámetro para la selección de cuerpo académico y del equipo de investigación. Se evidencia que en las investigaciones referentes a la GR está presente esa red de colaboración, en la que diversos autoresprincipalmente los que más han producido - emplean esa estrategia en su hacer científico.

La diversidad epistemológica en el hacer científico sobre GR se manifiesta en las temáticas planteadas y clasificadas, de acuerdo con lo señalado en siguiente tabla:

Tabla 5

Cantidad de artículos por ejes temáticos: Sistematizados por los autores.

\begin{tabular}{ccc}
\hline TEMÁTICA DEL ARTíCULO & TOTAL & $\%$ \\
\hline Entrenamiento & 20 & $41,67 \%$ \\
\hline Salud & 4 & $8,33 \%$ \\
\hline Iniciación deportiva y categorías de base & 4 & $8,33 \%$ \\
\hline Aspectos nutricionales & 4 & $8,33 \%$ \\
\hline Salud/entrenamiento & 4 & $8,33 \%$ \\
\hline Aspectos psicológicos & 4 & $8,33 \%$ \\
\hline Aspectos educacionales & 3 & $6,25 \%$ \\
\hline Aspectos sociales, culturales e históricos & 3 & $6,25 \%$ \\
\hline Aspectos psicológicos/ entrenamiento & 2 & $4,17 \%$ \\
\hline Suma general & 48 & $100,00 \%$ \\
\hline
\end{tabular}


El eje Entrenamiento fue el más recurrente, con un total de 20 artículos $(41,67 \%)$. (8,33\%), Iniciación Deportiva y Categorías de Base (8,33\%), Aspectos Nutricionales $(8,33 \%)$ y artículos clasificados en Salud / Entrenamiento (8,33\%) cada uno con cuatro artículos, intensifican la preeminencia epistemológica de las Ciencias Naturales, totalizando el 74,99\%. Las demás temáticas: Aspectos Psicológicos que contienen cuatro manuscritos (8,33\%), Aspectos Educacionales con tres (6,25\%), Aspectos Sociales, Culturales e Históricos también con tres escritos (6,25\%) y Aspectos psicológicos / Entrenamiento presentando dos trabajos (4,17\%), los que contemplan especificidades de la psicología a favor de la mejora del desempeño. Al total, se suma el 25\% de la producción encontrada.

En el análisis de los datos, es perceptible la inclinación de los estudios a las Ciencias Naturales, ya que, en revistas del área de Educación Física existe una tendencia de publicaciones que están ligadas a las vertientes biológicas, afirmación constatada en estudios anteriores de Manoel y Carvalho (2011) y Rigo, Ribeiro y Hallal (2011). La predisposición concuerda con la tradición epistemológica del área de Educación Física próxima a las Ciencias Biológicas, corroborando con el número superior de investigadores de esa área y, consecuentemente, con la superioridad en número de revistas y publicaciones en relación a las Ciencias Humanas.

El eje Entrenamiento presenta enfoques acerca de la preparación física y la aptitud física de las atletas de Gimnasia Rítmica. Trata de las características y cualidades físicas/biológicas/fisiológicas de las atletas, los análisis de equilibrio, maduración biológica y métodos de entrenamiento, sobre la influencia de la flexibilidad y del entrenamiento de fuerza en la ejecución de los movimientos característicos de la modalidad, y analiza la promoción de la calidad artística en la GR tanto en el entrenamiento de alto desempeño como en la práctica escolar. De este modo, en su totalidad se observó que, en la categoría de entrenamiento, la frecuencia de análisis acerca de los ejercicios para fuerza y flexibilidad fueron evidentes, además de constar la propuesta de protocolo o metodología en las encuestas para el tipo de entrenamiento.

Por ejemplo, se destaca el estudio de Ramos y Velásquez (2006), donde los autores elaboraron un sistema de orientaciones metodológicas para la preparación física (fuerza) específica para la modalidad de la GR, compuesto por tres grupos fundamentales de ejercicios: preparación general, con el circuito como principal ejemplo; los ejercicios auxiliares; y los específicos. En el estudio se especifican los beneficios del entrenamiento de fuerza, además de proporcionar subsidio para realizar la preparación $y$, de esa manera, demostrar cuán eficaz fue su protocolo. Para el entrenamiento de flexibilidad, el estudio de Karloh et al. (2010) realizó pruebas en las que el Grupo 1, al entrenar con el método Mulligan (Long Leg Traction) obtuvo ganancias estadísticamente 
significativas superiores a los del Grupo 2, que usó el estiramiento estático. Con el fin de complementar y reafirmar la importancia que algunos estudios que componen ese eje dan al entrenamiento de fuerza y flexibilidad, Silva et al (2016), con una intervención compuesta por ejercicios específicos, concluyeron que el trabajo específico de fuerza y flexibilidad puede influir de manera positiva en el entrenamiento de GR, sobre todo en los saltos, pues demandan una buena elevación del cuerpo, además de alcanzar amplitud de movimiento en el período de vuelo. Por lo tanto, se percibe una propensión de los artículos a verificar posibles relaciones de la mejora de determinadas capacidades físicas o habilidades motoras en el desempeño de practicantes de la modalidad.

Esos 20 artículos se encuentran en las siguientes revistas: Fitness \& Performance Journal (Penedo, Beltrão \& Nunes, 2006; Menezes \& Fernandes Filho, 2006) Ra Ximhai (Mena-Ramos \& Álvarez-Velásquez, 2006) Revista Brasileña de Cineantropometría y Desarrollo Humano (Karloh, Petry, Kraeski, Matias \& Frutuoso, 2010), Archivos de Ciencias de la Salud de la Unipar (Gonçalves, Barros Filho \& Gonçalves, 2010), Motricidad. European Journal of Human Movement (Vernetta, Fernández, López-Bedoya, Gómez-Landero \& Oña, 2011), Revista Brasileña de Ciencia y Movimiento (Pinto Júnior, Sousa, Gaya \& Alves, 2012; Del Vecchio, Primeira, Silva, Dall'agnol \& Galliano, 2014), Revista de Salud Pública (Souza Menezes, Novaes \& Fernandes-Filho, 2012), Revista Brasileña de Medicina del Deporte (Perin, Ulbricht, Ricieri \& Neves, 2012; Shigaki et al., 2013), Journal of Human Sport and Exercise (Bobo-Arce \& Méndez-Rial, 2013), Revista Brasileña de Educación Física y Deporte (Goulart et al 2014; Santos, Lebre \& Carvalho, 2016; Loquet, 2016), International Journal of Morphology (Menezes, Novaes \& Fernandes-Filho, 2014; Arriaza et al 2016), Revista Andaluza de Medicina del Deporte (Guimaraes-Ribeiro, Hernández-Suárez, Rodríguez-Ruiz \& García-Manso, 2015), Acta Scientarum Health Sciences (Boligon, Deprá, \& Rinaldi, 2015) y Revista Salud y Investigación (Silva, Oliveira, Leme, Nascimento Júnior \& Anversa, 2016).

Los cuatro escritos clasificados en el eje Salud cuentan con estudios sobre: evaluación física funcional, historial y análisis de lesiones, desequilibrios hormonales en atletas de la GR y análisis de los aspectos nutricionales, antropométricos y psicológicos en gimnastas de la modalidad. Las lesiones más recurrentes analizadas fueron musculares y tendinosas, con el predominio de dolores de espalda - aspecto que requiere consideración para mejorar la prevención. Por lo tanto, en lo que se refiere a este eje temático, se nota una tendencia por parte de los investigadores en utilizar la modalidad aquí estudiada como herramienta para análisis de factores que no 
concuerdan directamente con el momento del entrenamiento y de la competición, pero con las condiciones que regulan el mantenimiento físico del practicante.

Dichas investigaciones se encuentran en las revistas: Unopar Científica Ciencias Biológicas y de la Salud (Guiotte et al., 2012), Revista Brasileña de Ciencias del Deporte (Coelho, Simões \& Lunz, 2015), Revista Andaluza de Medicina del Deporte (Vernetta, Montosa \& Lopez-Bedoya, 2016), Nutrición Hospitalaria (San Mauro Martin, Cevallos, Pina Ordunez \& Garicano Vilar, 2016).

Con cuatro artículos, la clasificación Iniciación Deportiva y Categoría de Base contempla los siguientes temas: estudio de revisión sobre la detección y selección de talentos deportivos en la GR, la comparación de métodos en el aprendizaje de una habilidad específica de la modalidad y efectos de la retroalimentación en el entrenamiento y, por último, la investigación de la influencia de los programas de iniciación deportiva en el desempeño y desarrollo de habilidad para la maestría. Lanaro Filho e Böhme (2001) tratan sobre la necesidad de caracterizar el caso de las gimnastas de alto rendimiento en diferentes grupos de edad, con el fin de obtener indicadores que puedan servir de referencias para estimar cuáles son las reales posibilidades de que las gimnastas principiantes alcancen el alto nivel de desempeño deportivo - y no más apoyarse en elecciones subjetivas sin base científica. En este eje temático, se encontró una predisposición por parte de los investigadores a utilizar la iniciación deportiva para detectar talentos, para determinar la evolución técnica, táctica y física de los jóvenes practicantes en las edades más variadas. Estos estudios se encuentran en la Revista Brasileña de Educación Física y Deporte (Lanço Filho \& Böhme, 2001; Lemos, Chiviacowsky, Avila \& Drews, 2013), Revista Brasileña de Ciencia y Movimiento (Caçola \& Ladewig, 2007) y Revista de Educación Física/ UEM (Sampaio \& Valentini, 2015).

La temática Aspectos Nutricionales, también con un total de cuatro manuscritos, explora los estudios predominantes sobre los trastornos alimentarios y sus consecuencias, la influencia en el crecimiento físico de las gimnastas, la distorsión de la imagen corporal en las competiciones, el estudio de la composición corporal y el riesgo de trastorno de conducta alimentaria en los atletas del estado de Carabobo (Venezuela). El enfoque diferencial fue sobre el uso de la bebida artesanal (casera) como una forma de suplementación en los atletas de GR.

Debido a que es una modalidad en la cual su eficiencia depende, de cierta manera, de un bajo peso, muchos atletas terminan desarrollando un desequilibrio entre la ingesta de nutrientes y el ejercicio físico, además del posible riesgo de un trastorno de la conducta alimentaria. Aquí identificamos que es unánime entre los interesados en estudiar el eje respectivo la preocupación por el impacto que generan los hábitos nutricionales en el desarrollo y la composición corporal de los gimnastas. Los artículos 
fueron impresos en la Revista Brasileña de Medicina Deportiva (Vieira, Vieira, Amorim, Amorim \& Rocha, 2009), Motriz (Vieira, Vieira, Amorim y Amorim \& Rocha, 2009), Salus (Palacios De Espig \& Jaeger, 2016) y en la Revista Brasileña de Ciencias del Deporte (Costa et al., 2017).

Los artículos encuadrados en los ejes de Salud y Entrenamiento obtuvieron esta clasificación porque presentaron temas con el propósito de mejorar el rendimiento, pero con un sesgo de tratamiento y prevención de lesiones futuras. Contiene los manuscritos que se refieren al análisis postural de los atletas como medida preventiva y de ayuda para un mejor desarrollo de los movimientos e importante como medida profiláctica para identificar las posibles desalineaciones posturales y prevenir cambios posturales que puedan ocurrir, análisis de equilibrio y cómo se pueden evitar las lesiones resultantes de la demanda de cambios posicionales de la extremidad inferior, así como la investigación sobre el entrenamiento propioceptivo y sus efectos en la prevención de lesiones de gimnastas. En general, se observa que los investigadores interesados en estas discusiones utilizan factores concernientes a la salud como medios para mejorar el rendimiento durante el entrenamiento y las competiciones. Esos escritos se encuentran en la Revista Brasileña de Medicina Deportiva (Bosso \& Golias, 2012; Lamb et al., 2014), Revista Brasileña de Ciencia y Movimiento (Golias, 2012) y Conscientiae Saúde (Hirata \& Oliveira, 2015).

La temática Aspectos Psicológicos incluye artículos que tratan de factores motivacionales compuestos por el gusto por la práctica, el establecimiento de metas y factores estresantes pertinentes al bajo rendimiento académico, los desacuerdos y conflictos, la falta de tiempo para la diversión en el entorno competitivo y en el arbitraje. La participación y la influencia de los padres en la carrera deportiva, y la evaluación y el análisis de las habilidades psicológicas presentes en atletas de gimnasia rítmica también formaron parte del alcance. Se observó que concerniente a este tema hubo una tendencia por parte de los investigadores en investigar cómo ciertas características psicológicas influyen en la formación de los profesionales de esta modalidad. Dichos textos pertenecen a las siguientes revistas: Acta Scientarum Health Sciences (Vieira, Botti \& Vieira, 2005), Revista Iberoamericana de Psicología del Ejercicio y el Deporte (Sánchez, Márquez \&, 2010), Pensar la Práctica (Nakashima, Nascimento Junior \& Vieira, 2012), Revista de la Educación Física/UEM (Debien, Noce, Debien \& Costa, 2014).

Los Aspectos educativos abarcan temas relacionados con la formación profesional de las técnicas/profesoras de GR y la gimnasia rítmica como contenido académico. En general, estos artículos buscan enfatizar la importancia de formar buenos profesionales para el desarrollo deportivo, así como la necesidad de un mayor 
contacto entre los estudiantes con la modalidad en el contexto académico. Los estudios se encuentran en las páginas de la revista Motriz (Belão, Machado y Mori, 2009), Pensar la Práctica (Oliveira y Porpino, 2010), Revista Internacional de Medicina y Ciencias de la Actividad Física y el Deporte (Amengual y Lleixà, 2011).

Los tres artículos con un enfoque temático en Aspectos sociales, culturales e históricos traen el análisis de los códigos de calificación de GR en sí mismos, evidenciando cambios históricos en técnicas y aspectos artísticos. Además, se presenta un estudio que investiga las relaciones entre la belleza, el sufrimiento y el dolor en la gimnasia rítmica, desde una perspectiva filosófica. Se hace evidente que el trabajo de este eje toma en consideración el comprender cómo la construcción histórica de esta modalidad ha tenido lugar a través de varias rutas socioculturales. Los escritos están vinculados a la Revista de Investigación (Mejías y María, 2013), Holos (Cavalcanti y Porpino, 2015) y la Revista Brasileña de Educación Física y Deporte (Toledo y Antualpa, 2016).

Publicados en la revista Thinking the Practice (Fontana, Barbosa, Balbinotti \& Balbinotti, 2013) y en la Revista de Psicología del Deporte (Álvarez, Falco, Estevan, Molina-García \& Castillo, 2013), los artículos relacionados con ambos Aspectos psicológicos y Entrenamiento tratan temas relacionados con las contribuciones de la motivación al rendimiento y el análisis de la intervención de psicólogos en un equipo de gimnasia rítmica. Estos estudios buscan relacionar los posibles beneficios de las intervenciones motivacionales con el rendimiento durante el entrenamiento de los practicantes de Gimnasia Rítmica.

Si bien las encuestas son predominantes en el eje de Entrenamiento, la diversidad en el material analizado es indiscutible. Esto es debido a la contribución de numerosos investigadores de Educación Física que transitan en otras áreas y académicos de distintas áreas que se dedican a explorar aspectos relacionados con el deporte.

\section{Consideraciones Finales}

El presente artículo buscó delinear la producción científica sobre la Gimnasia Rítmica presente en las publicaciones periódicas de América Latina, el Caribe y los Países lbéricos. Ese conjunto de países se escogió debido al creciente desarrollo de la modalidad. Es pertinente realizar el estudio en dicha área geográfica porque, en comparación con los países hegemónicos en la modalidad (Rusia, Bulgaria, Ucrania, Bielorrusia e Italia), las naciones latinoamericanas, caribeñas e ibéricas están ganando gradualmente un espacio en el escenario competitivo. En ese sentido, el análisis de 
cómo la gimnasia rítmica se ha estado desarrollando en el universo científico en la delimitación espacial respectiva se vuelve importante para poder fomentar mayores discusiones sobre la práctica, contribuyendo directamente al incentivo para los investigadores interesados en este objeto inicien nuevas investigaciones.

Con esto, se buscó identificar cómo los investigadores estudiaron la modalidad en la periodicidad delimitada entre los años 2001 y 2017. Es pertinente advertir sobre las limitaciones prácticas del estudio presentado, debido a que solo se utilizaron artículos publicados en revistas latinoamericanas, caribeñas e iberoamericanas. Sobre todo, si consideramos a la comunidad científica en un contexto donde la internacionalización es indiscutible y que el uso de bases de datos más amplias alteraría la metodología y los resultados de la investigación. Por lo tanto, se entiende que un nuevo desenlace para investigaciones a este respecto serían posibles comparaciones entre los hallazgos sobre la modalidad en las localidades más distintas, así como una mirada a otros productos, como disertaciones y tesis, por ejemplo. Otra recomendación se refiere a la elaboración de nuevas categorías temáticas, ya que al fin y al cabo se utilizaron las perspectivas de otros investigadores para el presente; por tanto, se entiende que, con nuevas perspectivas, pueden surgir nuevos ejes temáticos.

Finalmente, el estudio encontró 48 artículos publicados en 27 revistas diferentes, de las cuales 17 son brasileñas (con un total de 36 artículos), siete españolas (con ocho artículos), una chilena con dos textos y las revistas colombianas y mexicanas con un artículo cada una. De estos, predominan las publicaciones de la Revista Brasileña de Educación Física y Deporte con seis manuscritos, la Revista Brasileña de Medicina del Deporte con cinco, la Revista Brasileña de Ciencia y Movimiento con cuatro y Pensar la Práctica que contiene tres escritos. Las otras 26 revistas tenían uno o dos artículos cada una. La temática que más despertó el interés de los investigadores está relacionada con el Entrenamiento, que corresponde al $41,67 \%$ de toda la producción científica encontrada sobre la GR.

A pesar del predominio de dicho eje, se evidencia la pluralidad epistemológica en el hacer ciencia sobre la Gimnasia Rítmica en Latinoamérica, el Caribe y los Países Ibéricos, ya que fue contemplada por diversos enfoques epistemológicos. Por lo tanto, es un escenario propicio para un área que pretende consolidarse científicamente, siendo necesario el crecimiento de estudios apoyados en las otras formas de acercarse al deporte, ascendiendo de forma cuantitativa y cualitativa a estas producciones. Se señala aquí la importancia práctica de este trabajo; después de todo, se cree que con él pueden emerger nuevos estudios en los ejes temáticos más variados, incluso con perspectivas epistemológicas diferentes de las encontradas en estos estudios. 
En relación con el modelo de producción de los artículos, se verificó la existencia de la red de colaboración de autores a través del número de autores/instituciones que cooperaron entre sí. Esta tendencia en estudios previos de García et al. (2010) ha sido evidenciada como próspera para los investigadores y para el campo científico. En lo tocante al país de la institución donde los autores estaban vinculados, Brasil presentó superioridad, con la suma de 35 instituciones. Luego, España con ocho instituciones y Portugal y Venezuela con dos cada uno y, apenas con una institución asociada están: Francia, Cuba, México, Canadá, Chile y los Estados Unidos. Se observa que el hecho de que la mayoría de los estudios son de Brasil y España puede tener una relación directa de que estos son los países dentro de la delimitación espacial con mayor prominencia en los resultados de las competiciones mundiales. De esta forma, se puede inferir que el universo de la investigación y el avance competitivo está relacionado, existiendo en el área el inicio de una aproximación del hacer científico con el profesional.

Por lo tanto, se considera que el presente estudio puede presentar una contribución a la comunidad académica, señalando el modus operandi relacionado con la producción del conocimiento sobre la Gimnasia Rítmica, alentando una mayor producción sobre la temática en sus diversos aspectos investigables. Se concluye que este estudio ofrece subsidios metodológicos prácticos para ampliar la comprensión sobre el marco actual de la producción del conocimiento sobre la GR en Latinoamérica, el Caribe y los Países Ibéricos y los datos presentados, así como la comprensión de ellos, lo que puede contribuir como fomento del conocimiento. Después de todo, se entiende que con esta escritura se puede instigar a los interesados en el tema para que inicien nuevos estudios, principalmente en lo que concierne a las temáticas menos incidentes. Además, se señala la necesidad práctica, como afirma Lourenço (2003), de más investigaciones que puedan contribuir de manera efectiva al trabajo de quienes a diario hacen que la modalidad suceda.

\section{Referencias}

Álvarez, O., Falco, C., Estevan, I., Molina-García, J., \& Castillo, I. (2013). Intervención psicológica en un equipo de gimnasia rítmica deportiva: Estudio de un caso. Revista de Psicología del Deporte, 22(2), 395-401. Recuperado de http://www.redalyc.org/articulo.oa?id=235128058008

Amengual, M., \& Lleixà, T. (2011). La creatividad motriz en gimnasia rítmica deportiva en edad escolar. International Journal of Medicine and Science of Physical Activity and Sport, 11(43), 548-563. Recuperado de http://www.redalyc.org/articulo.oa?id=54222177007 
Arriaza, E., Rodríguez, C., Carrasco, C., Mardones, C., Niedmann, L., \& López-Fuenzalida, A. (2016). Anthropometric characteristics of elite rhythmic gymnasts. International Journal of Morphology, 34(1), 17-22. Recuperado de https://www.researchgate.net/publication/301205196 Anthropometric Characteristics of Elite Rhythmic Gymnasts

Belão, M., Machado, L. P., \& Mori, P. M. M. (2009). A formação profissional das técnicas de ginástica rítmica. Motriz: revista de educação física (Impresso), 15(1), 61-68. Recuperado de http://www.periodicos.rc.biblioteca.unesp.br/index.php/motriz/article/view/2139/2284

Bobo-Arce, M. \& Rial, B. M. (2013). Determinants of competitive performance in rhythmic gymnastics. A review. Journal of Human Sport and Exercise, 8(3), 711-727. Recuperado de http://www.redalyc.org/articulo.oa?id=301028375009

Boligon, L., Deprá, P. P., \& Rinaldi, I. P. B. (2015). Influence of flexibility in the ex ecution of movements in rhythmic gymnastics. Acta Scientiarum. Health Sciences, 37(2), 141-145. Recuperado de http://www.redalyc.org/articulo.oa?id=307242823005

Bosso, L. R., \& Golias, A. R. C. (2012). A postura de atletas de ginástica rítmica: análise através da fotometria. Revista Brasileira de Medicina do Esporte, 18(5), 333-337. Recuperado de http://www.scielo.br/scielo.php?script=sci arttext\&pid=S1517-86922012000500010

Caçola, P. M., \& Ladewig, I. (2007). Comparação entre as práticas em partes e como um todo e a utilização de dicas na aprendizagem de uma habilidade da ginástica rítmica. Revista Brasileira de Ciência e Movimento, 15(4), 79-86. Recuperado de http://www.metaproducoes.com.br/artigos/treinamento/8.pdf

Cavalcanti, L. M. B., \& Porpino, K. O. (2015). O sofrimento e a dor como constituintes da beleza esportiva: reflexões para a educação. Holos, 5 . Recuperado de http://www.redalyc.org/pdf/4815/481547288032.pdf

Coelho, S. M. H., Simões, R. D., \& Lunz, W. (2015). Desequilíbrio hormonal e disfunção menstrual em atletas de ginástica rítmica. Revista Brasileira de Ciências do Esporte, 37(3), 222-229. Recuperado de http://www.scielo.br/pdf/rbce/v37n3/01013289-rbce-37-03-0222.pdf

Costa, T. A., Gonçalves, H. R., Anschau, F. R., Viaro, L. F., Borgheti, R., dos Santos, F. B., \& Borges, J. H. (2017). Suplementação com bebida artesanal que contém carboidrato em atletas da ginástica rítmica. Revista Brasileira de Ciências do Esporte, 39(2), 115-122. Recuperado de http://www.scielo.br/pdf/rbce/v39n2/0101-3289-rbce-39-02-0115.pdf

Debien, P. B., Noce, F., Debien, J. B. P., \& da Costa, V. T. (2014). O estresse na arbitragem de ginástica rítmica: uma revisão sistemática. Journal of Physical Education, 25(3), 489500. Recuperado de http://www.scielo.br/scielo.php?pid=S1983$\underline{30832014000300489 \& \text { script }=\text { sci abstract }}$

Del Vecchio, F. B., Primeira, M., Silva, H. C. D., Dall'agnol, C., \& Galliano, L. M. (2014). Nível de aptidão física de atletas de ginástica rítmica: Comparações entre categorias etárias. Revista brasileira de Ciência e Movimento, 22(3), 5-13. Recuperado de https://portalrevistas.ucb.br/index.php/RBCM/article/view/4444

Fontana, P.; Barbosa, M.; Balbinotti, M.; Balbinotti, C. (2013) Promoting artistic quality in rhythmic gymnastics: a didactic analysis from high performance to school practice. 
Pensar a Prática, 16, 320-618. Recuperado de http://www.scielo.br/scielo.php?script=sci arttext\&pid=S1807-55092016000100145

Garcia, C. C., Martrucelli, C. R. N., Rossilho, M. D. M. F., \& Denardin, O. V. P. (2010). Autoria em artigos científicos: os novos desafios. Revista Brasileira de Cirurgia Cardiovascular/Brazilian Journal of Cardiovascular Surgery, 25(4), 559-567. Recuperado de http://www.scielo.br/pdf/rbccv/v25n4/v25n4a21

Golias, A. R. C. (2012). Relação entre instabilidade do joelho e alterações posicionais do membro inferior de atletas de Ginástica Rítmica. Revista Brasileira de Ciência e Movimento, 20(2), 52-60. Recuperado em https://portalrevistas.ucb.br/index.php/RBCM/article/view/3153

Gonçalves, L. A. P., de Azevedo Barros Filho, A., \& Gonçalves, H. R. (2010). Características antropométricas de atletas de ginástica rítmica. Arquivos de Ciências da Saúde da UNIPAR, 14(1), 17-25. Recuperado em http://revistas.unipar.br/index.php/saude/article/view/3399

Goulart, N. B. A., Dias, C. P., Lemos, F. D. A., Oliva, J. C., Lanferdini, F. J., \& Vaz, M. A. (2014). Evaluation of passive plantarflexion torque and active dorsiflexion torque in rhythmic gymnasts and nonathletes. Revista Brasileira de Educação Física e Esporte, 28(3), 371376. Recuperado em http://www.scielo.br/scielo.php?script=sci arttext\&pid=S1807$\underline{55092014000300371}$

Guiotte, V. A., Roque, C. F., de Oliveira, M. R., de Oliveira Gil, A. W., Rabello, L. M., Nobre dos Santos, E. V., ... \& da Silva, R. A. (2012). Avaliação físico-Funcional de Atletas da Ginástica Ritmica: História de Lesoes e Estabilidade Postural. UNOPAR Científica. Ciências Biológicas e da Saúde, 14(4), 217-220. Recuperado de http://www.pgsskroton.com.br/seer/index.php/JHealthSci/article/view/832

Hirata, A. C. D. S., \& Oliveira, R. F. D. (2015). Protocolo de treinamento proprioceptivo para atletas de Ginástica Rítmica-GR. ConScientiae Saúde, 14(4), 634-640. Recuperado de http://www.redalyc.org/pdf/929/92945642015.pdf

Júnior, J. A. D. P., de Sousa, M. D. S. C., Gaya, A. C. A., \& Alves, J. V. D. M. H. (2012). Maturação biológica e desempenho físico de jovens atletas de ginástica rítmica. Revista Brasileira de Ciência e Movimento, 20(1), 88-96. Recuperado de https://portalrevistas.ucb.br/index.php/RBCM/article/view/2708

Karloh, M., Santos, R. P. D., Kraeski, M. H., Matias, T. S., \& Frutuoso, A. S. (2010). Alongamento estático versus conceito Mulligan-efeitos crônicos no treino de flexibilidade em ginastas. Rev Bras Cineantrop Desemp Hum, 12(3), 202-208. Recuperado de http://www.scielo.br/pdf//m/v23n4/a03v23n4

Lamb, M., Oliveira, P. D. D., Tano, S. S., Gil, A. W. D. O., Santos, E. V. N. D., Fernandes, K. B. P., \& Oliveira, R. F. D. (2014). Efeito do treinamento proprioceptivo no equilíbrio de atletas de ginástica rítmica. Revista Brasileira de Medicina do Esporte, 20(5), 379-382. Recuperado de http://www.scielo.br/pdf/rbme/v20n5/1517-8692-rbme-20-05-00379.pdf

Lanaro Filho, P., \& Böhme, M. T. S. (2001). Deteç̧ão, seleção e promoção de talentos esportivos em ginástica rítmica desportiva: um estudo de revisão. Revista Paulista de Educação Física, 15(2), $141-153 . \quad$ Recuperado de http://www.revistas.usp.br/rpef/article/view/139898 
Lemos, A., Chiviacowsky, S., Ávila, L. T. G., \& Drews, R. (2013). Efeitos do" feedback" autocontrolado na aprendizagem do lançamento da bola da ginástica rítmica. Revista Brasileira de Educação Física e Esporte, 27(3), 485-492. Recuperado de http://www.scielo.br/scielo.php?pid=S180755092013000300014\&script=sci abstract\&tl $\underline{\mathrm{ng}=\mathrm{pt}}$

Leta, J., Cruz, C. H. B. (2003) A produção cientifica brasileira. In: Viotti, Eduardo Baumgratz; Macedo, Mariano de Matos (Org.). Indicadores de Ciência, Tecnologia e Inovação no Brasil. Campinas: Editora Unicamp,p. 121-168.

Loquet, M. (2016). Promoting artistic quality in rhythmic gymnastics: a didactic analysis from high performance to school practice. Revista Brasileira de Educação Física $e$ Esporte, 30(1), 145-158. Recuperado de http://www.scielo.br/scielo.php?script=sci arttext\&pid=S1807-55092016000100145

Lourenço, M. R. A. (2003) Ginástica Rítmica no Brasil: a (r)evolução de um esporte. 154 f. Dissertação. Universidade Metodista de Piracicaba, Piracicaba. Recuperado de http://cev.org.br/biblioteca/ginastica-ritmica-brasil-revolucao-um-esporte/

Lourenço, M. R. A. (2015). A seleção brasileira de conjuntos de ginástica rítmica: perfil de ginastas e treinadoras, estrutura técnica e administrativa e o habitus construído. $180 \mathrm{f}$. Tese. Universidade Estadual de Maringá, Maringá. Recuperado de http://nourau.uem.br/nou-rau/document/?code=vtls000220788

Manoel, E. D. J., \& Carvalho, Y. M. D. (2011). Pós-graduação na educação física brasileira: a atração (fatal) para a biodinâmica. Educação e Pesquisa, vol. 37, núm. 2, mayo-agosto, 2011, pp. 389-406 Universidade de São Paulo São Paulo, Brasil. Educação e Pesquisa, 37(2), 389-406. Recuperado de http://www.scielo.br/scielo.php?pid=S1517$97022011000200012 \&$ script $=$ sci abstract\&tlng=pt

Mejías, N., \& María, J. (2013). Cambios de la técnica corporal en la modalidad de Gimnasia Rítmica. Códigos de puntuación 1993-2005. Revista de Investigación, 37(79), 101-118. Recuperado de http://revistas.upel.edu.ve/index.php/revinvest/article/view/3084

Mena-Ramos, R., \& Álvarez-Velásquez, I. (2006). Orientaciones metodológicas para desarrollar la preparación física (fuerza) en las atletas de gimnasia rítmica. Ra Ximhai: revista científica de sociedad, cultura y desarrollo sostenible, 2(2), 515-532. Recuperado de http://www.redalyc.org/articulo.oa?id=46120212

Menezes, L. D. S., Novaes, J., \& Fernandes-Filho, J. (2012). Qualidades físicas de atletas e praticantes de Ginástica Rítmica pré e pós-púberes. Revista de Salud Pública, 14, 238247. Recuperado de http://www.redalyc.org/articulo.oa?id=42225409004

Menezes, L. S., Novaes, J.; Fernandes Filho, J. (2014). Somatotipo de atletas e praticantes de Ginástica Rítmica pré e pós-púberes. International Journal of Morphology , 32, 10251029. Recuperado de http://www.intjmorphol.com/wpcontent/uploads/2015/06/art 36 323.pdf

Nakashima, F. S., Nascimento Junior, J.R., \& Vieira, L. F. (2012). O papel dos pais na trajetória esportiva de atletas de ginástica rítmica. Pensar a Prática, 15(4).821-1113. Recuperado de https://www.revistas.ufg.br/fef/article/view/14380

Oliveira, G. M., \& Porpino, K. D. O. (2010). Ginástica rítmica e educação física escolar: perspectivas críticas em discussão. Pensar a Prática, 13(2),116. Recuperado de https://www.revistas.ufg.br/fef/article/view/8632 
Palacios de Espig, V., \& Sánchez Jaeger, A. (2016). Composición corporal y riesgo de trastorno de conducta alimentaria en atletas de gimnasia rítmica del Estado Carabobo. Salus, 20(3), 7-11. Recuperado de http://www.redalyc.org/pdf/3759/Resumenes/Resumen 375949531003 1.pdf

Penedo, T., Beltrao, F., \& Nunes, W. J. (2006). Análise da orientação espaço-temporal no desempenho motor competitivo em ginástica rítmica: Categorias pré-infantil e infantil. Fitness \& performance journal, (1), 44-49. Recuperado de http://www.fpjournal.org.br/painel/arquivos/7947\%20Orientaca0\%20na\%20GRD\%20Rev\%201\%202006\%20Portugues.pdf

Perin, A., Ulbricht, L., da Vinha Ricieri, D., \& Neves, E. B. (2012). Utilização da biofotogrametria para a avaliação da flexibilidade de tronco. Revista Brasileira de Medicina do Esporte, 18(3), 176-180. Recuperado de http://www.scielo.br/scielo.php?script=sci arttext\&pid=S1517-86922012000300008

Ribeiro, D. G., Suárez, M. H., \& García-Manso, J. M. (2015). Efecto del entrenamiento sistemático de gimnasia rítmica sobre el control postural de niñas adolescentes. Revista andaluza de medicina del deporte, 8(2), 54-60. Recuperado em http://www.redalyc.org/articulo.oa?id=323338744002

Rigo, L. C., Ribeiro, G. M., \& Hallal, P. C. (2011). Unidade na diversidade: desafios para a Educação Física no século XXI. Revista Brasileira de Atividade Física \& Saúde, 16(4), 339-345. Recuperado de rbafs.org.br/RBAFS/article/download/631/661/

Sampaio, D. F., \& Valentini, N. C. (2015). Iniciação esportiva em ginástica rítmica: abordagens tradicional e o clima de motivação para a maestria. Journal of Physical Education, 26(1), 1-10. Recuperado

http://www.periodicos.uem.br/ojs/index.php/RevEducFis/article/view/22382

San Mauro Martín, I., Cevallos, V., Pina Ordúñez, D., \& Garicano Vilar, E. (2016). Aspectos nutricionales, antropométricos y psicológicos en gimnasia rítmica. Nutrición Hospitalaria, 33(4), 865-871. Recuperado de http://scielo.isciii.es/scielo.php?script=sci arttext\&pid=S0212-16112016000400016

Sánchez, J. C. J., Márquez, J. C., \& Peralto, E. L. (2010). Evaluación y análisis de habilidades psicológicas relacionadas con el rendimiento deportivo en gimnastas de rítmica. Revista Iberoamericana de Psicología del Ejercicio y el Deporte, 5(1), 15-28. Recuperado de http://www.redalyc.org/articulo.oa?id=311126267002

Santos, A. B., Lebre, E., \& Carvalho, L. Á. (2016). Explosive power of lower limbs in rhythmic gymnastics athletes in different competitive levels. Revista Brasileira de Educação Física e Esporte, 30(1), 41-50. Recuperado de http://www.scielo.br/scielo.php?script=sci arttext\&pid=S1807-55092016000100041

Santos, E. V. N., Lourenço, M. R. A., \& Gaio, R. (2010). Composição coreográfica em ginástica rítmica: do compreender ao fazer. Jundiaí: Fontoura, 1, 127. Recuperado de https://periodicos.ufsc.br/index.php/motrivivencia/article/viewFile/2175$\underline{8042.2010 v 22 n 35 p 279 / 18096}$

Shigaki, L., Rabello, L. M., Camargo, M. Z., da Costa Santos, V. B., de Oliveira Gil, A. W., de Oliveira, M. R., \& Macedo, C. D. S. G. (2013). Análise comparativa do equilíbrio unipodal de atletas de ginástica rítmica. Revista Brasileira de Medicina do Esporte, 19(2), 104107. Recuperado de http://www.scielo.br/scielo.php?script=sci arttext\&pid=S1517$\underline{86922013000200006}$ 
Silva, J. M., de Oliveira, D. V., Leme, D. E. C., do Nascimento Júnior, J. R. A., \& Anversa, A. L. B. (2016). Influência do treinamento de flexibilidade e força muscular em atletas de ginástica rítmica. Saúde e Pesquisa, 9(2), 325-331. Recuperado de http://periodicos.unicesumar.edu.br/index.php/saudpesq/article/view/5217

Souza, D. L., Moraes, M., \& Moreira, T. S. (2016). O perfil da produção científica online em português relacionada às modalidades olímpicas e paralímpicas. Movimento, 22(4), 1105-1120. Recuperado de https://seer.ufrgs.br/Movimento/article/view/64591

Toledo, E. D., \& Antualpa, K. (2016). The appreciation of artistic aspects of the Code of Points in rhythmic gymnastics: an analysis of the last three decades. Revista Brasileira de Educação Física e Esporte, 30(1), 119-131. Recuperado de http://www.scielo.br/scielo.php?script=sci arttext\&pid=S1807-55092016000100119

Vernetta, M., Fernández, E., Bedoya, J. L., Landero, A. G., \& Oña, A. (2011). Estudio relacional entre el perfil morfológico y estima corporal en la selección andaluza de gimnasia rítmica deportiva. European Journal of Human Movement, (26), 77-92. Recuperado de http://www.redalyc.org/articulo.oa?id=274219446006

Vernetta, M., Montosa, I., \& López-Bedoya, J. (2016). Análisis de las lesiones deportivas en jóvenes practicantes de gimnasia rítmica de competición en categoría infantil. Revista Andaluza de Medicina del Deporte, 9(3), 105-109. Recuperado de http://scielo.isciii.es/scielo.php?script=sci abstract\&pid=S1888-75462016000300002

Vieira, L. F., Botti, M., \& Vieira, J. L. L. (2005). Ginástica rítmica: análise dos fatores competitivos motivadores e estressantes da Seleção Brasileira Juvenil. Acta Scientiarum. Health Sciences, 27(2), 207-215. Recuperado de http://periodicos.uem.br/ojs/index.php/ActaSciHealthSci/article/view/1419

Vieira, J. L. L., Amorim, H. Z., Vieira, L. F., Amorim, A. C., \& da Rocha, P. G. M. (2009). Distúrbios de atitudes alimentares e distorção da imagem corporal no contexto competitivo da ginástica rítmica Eating disorders and body image distortion in the rhythmic gymnastics competitive context. Revista Brasileira de Medicina do Esporte, 15(6), 410-414. Recuperado de http://www.scielo.br/scielo.php?script=sci arttext\&pid=S1517-86922009000700001

Vieira, L. F., Amorim, H. Z., Amorim, A. C., Rocha, P. G. M. D., \& Vieira, J. L. L. (2009). Distúrbios de atitudes alimentares e sua relação com o crescimento físico de atletas paranaenses de ginástica rítmica. Motriz : revista de educação física (Impresso), 15(3), 552-561. 